\title{
A MODEL FOR COUPLED BELOUSOV-ZHABOTINSKY OSCILLATORS WITH DELAY
}

\author{
*Giovanni Pagano ${ }^{1}$, Marcello Antonio Budroni ${ }^{2}$, Raffaele D’Ambrosio ${ }^{3}$, Dajana Conte ${ }^{1}$, \\ Ali Abou Hassan ${ }^{4}$, Sandra Ristori ${ }^{5}$, Federico Rossi ${ }^{6}$ and Beatrice Paternoster ${ }^{1}$ \\ ${ }^{1}$ Department of Mathematics, University of Salerno \\ e-mail: \{gpagano, dajconte, beapat $\} @$ unisa.it \\ 2 Department of Chemistry and Pharmacy, University of Sassari \\ e-mail: mabudroni@uniss.it \\ ${ }^{3}$ Department of Engineering and Computer Science and Mathematics, University of L'Aquila \\ e-mail: raffaele.dambrosio@univaq.it \\ ${ }^{4}$ Physico-Chemistry Laboratory of Electrolytes and Nanosystems Interfaciaux, Sorbonne University \\ e-mail: ali.abou_hassan@sorbonne-universite.fr \\ ${ }^{5}$ Department of Chemistry 'Ugo Schiff', University of Firenze \\ e-mail: sandra.ristori@unifi.it \\ ${ }^{6}$ Department of Physical Science, Earth and Environment, University of Siena \\ e-mail: federico.rossi2@unisi.it
}

Key words: Delay differential equations, Belousov-Zhabotinsky reaction

\begin{abstract}
The purpose of this work is to introduce time delays in an Oregonator-based model [11] to describe molecular communication in a system of coupled chemical oscillators [5]. With this approach we assume that the molecules responsible for communication (signal molecules) take a non-zero time $\tau$ to cross the barrier (phospholipid membrane) which physically separate the oscillators. In particular, here we present numerical results and we discuss and highlight the differences between delayed and non-delayed models.
\end{abstract}

\section{INTRODUCTION}

Networks of confined and relatively simple chemical oscillators are being used to model complex biological processes, such as cell-cell communication, neuronal signal transmission and passive diffusion across plasma membranes [26, 27]. From an experimental point of view, the confinement of the Belousov-Zhabotinsky (BZ) reaction [3, 28, 29] in various dispersed systems [10], revealed to be a valid tool to understand universal synchronisation behaviours, when the network communication is mediated by either activatory or inhibitory signals. The BZ reaction is one of the most famous example of selforganised chemical system, which can exhibit several dynamical regimes: periodic, aperiodic and chaotic oscillations [18, 20], autocatalysis and bistability [24], Turing structures and pattern formation [4, 19]. In 
Giovanni Pagano, Marcello Antonio Budroni, Raffaele D’Ambrosio, Dajana Conte, Ali Abou Hassan, Sandra Ristori, Federico Rossi and Beatrice Paternoster

the last years, especially thanks to microfluidic techniques, it was possible to assess the impact of several factors on the global dynamics of BZ networks: arrangement geometry (arrays, squares, etc.), messenger molecules (inhibitors/activators) and type of confinement (surfactants, phospholipids, oils, etc.) [26]. In this context, to model the oscillations of the coupled BZ oscillators, several variants of the Oregonator system [11] were employed, either coupled through simple mass-transfer (ODE systems) or through molecular diffusion (PDE systems). Being the Oregonator stiff, several studies were also devoted to its numerical resolution. D'Ambrosio et al. [7, 8], for example, have recently used adapted numerical methods to follow the apriori known qualitative behavior of the solution. To solve efficiently stiff ODEs systems of which we know certain properties of the solution, one can use the Exponential Fitting (EF) technique, thus constructing methods adapted to the problem [16]. By proceeding in this way, it is also possible to construct semi-implicit Runge-Kutta methods whose coefficients depend on the Jacobian matrix of the system $[9,13,14,15]$.

In this paper we introduce a modelling strategy based on delayed coupled ordinary differential equations (DDE) to understand whether this approach could improve some quantitative aspects for the description of BZ networks. To this aim, we started form an ODEs system proposed by Budroni et al. to describe the communication in a network of diffusively coupled BZ oscillators, confined in phospholipid-separated micro-compartments [5]. In this example, communication between successive oscillators is controlled by the exchange of membrane-permeable molecules acting as activator $\left(\mathrm{HBrO}_{2}\right)$ or inhibitor $\left(\mathrm{Br}_{2}\right)$, thus some properties of the membrane (composition, permeability, lamellarity, etc.) influence the crossing time of the messenger molecules. By inserting a delay time in one of the two coupling terms (in this case related to $\mathrm{Br}_{2}$ ) of the ODEs system, we can reproduce the effect of the membrane barriers and also

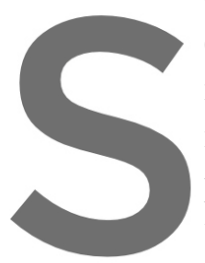
explore the parameter is also stiff, we used an integration. Our result: previous ODE model with experiments is yet to be attained. Interesting
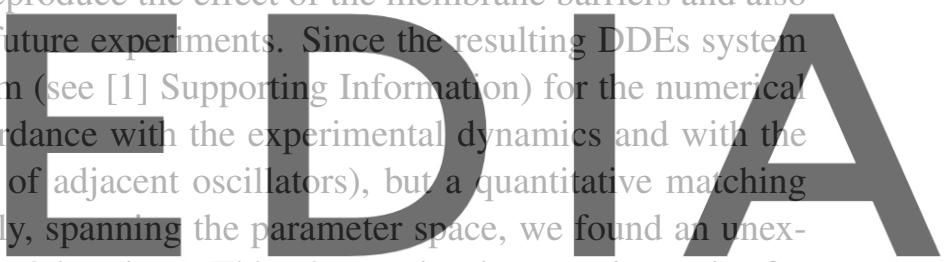

pected in-phase synchronisation scenario for large delay time. This observation is a starting point for

Register forther studies to guide future experiments. This work is structured as lollows: we will mist analyse, in Section 2, the ODEs model proposed by of the DDEs system. Next, in Section 3, we will show and analyse the numerical results of the DDEs system. Finally, in Section 4, some conclusions will be drawn and possible future implications will be discussed.

\section{THE MODEL WITH DELAY}

As aforementioned, arrays of phospholipid separated BZ micro-oscillators provide a relatively simple picture of the basic features responsible for the intercellular biological communication, when mediated by passive diffusion of activatory and/or inhibitory signal molecules. The mathematical description of such systems has been developed along the years starting from the classic scheme of the BZ reaction known as Oregonator $[11,25]$ and successively refined to include the effect of the membrane permeability [21], composition [27] and the simultaneous exchange of both activatory and inhibitory signals among micro-oscillators [5, 6].

In brief, the basic backbone of the kinetic scheme regulating the oscillatory regime of the BZ reaction 
Giovanni Pagano, Marcello Antonio Budroni, Raffaele D’Ambrosio, Dajana Conte, Ali Abou Hassan, Sandra Ristori, Federico Rossi and Beatrice Paternoster

consists in three main stages: inhibition (Process $A$ ), autocatalysis (Process $B$ ) and chemical clock reset (Process $C$ ), described by the following chemical processes:

$$
\begin{array}{ll}
\text { Process } A: & \mathrm{BrO}_{3}^{-}+\mathrm{Br}^{-}+2 \mathrm{H}^{+} \rightarrow \mathrm{HBrO}_{2}+\mathrm{HBrO} \\
\text { Process } B: & \mathrm{BrO}_{3}^{-}+\mathrm{HBrO}_{2}+3 \mathrm{H}^{+}+2 \mathrm{M}^{(r e d)} \rightarrow 2 \mathrm{HBrO}_{2}+2 \mathrm{M}^{(o x)} \\
\text { Process } C: & \mathrm{BrMA}+\mathrm{MA}+2 \mathrm{M}^{(o x)} \rightarrow \mathrm{f} \mathrm{Br}^{-}+2 \mathrm{M}^{(r e d)}
\end{array}
$$

The original set of Oregonator ordinary differential equations was derived from Processes $A-C$, here we report the latest version of the equations modified to simulate the behaviour of an arbitrary long array of BZ micro-oscillators [5, 6]:

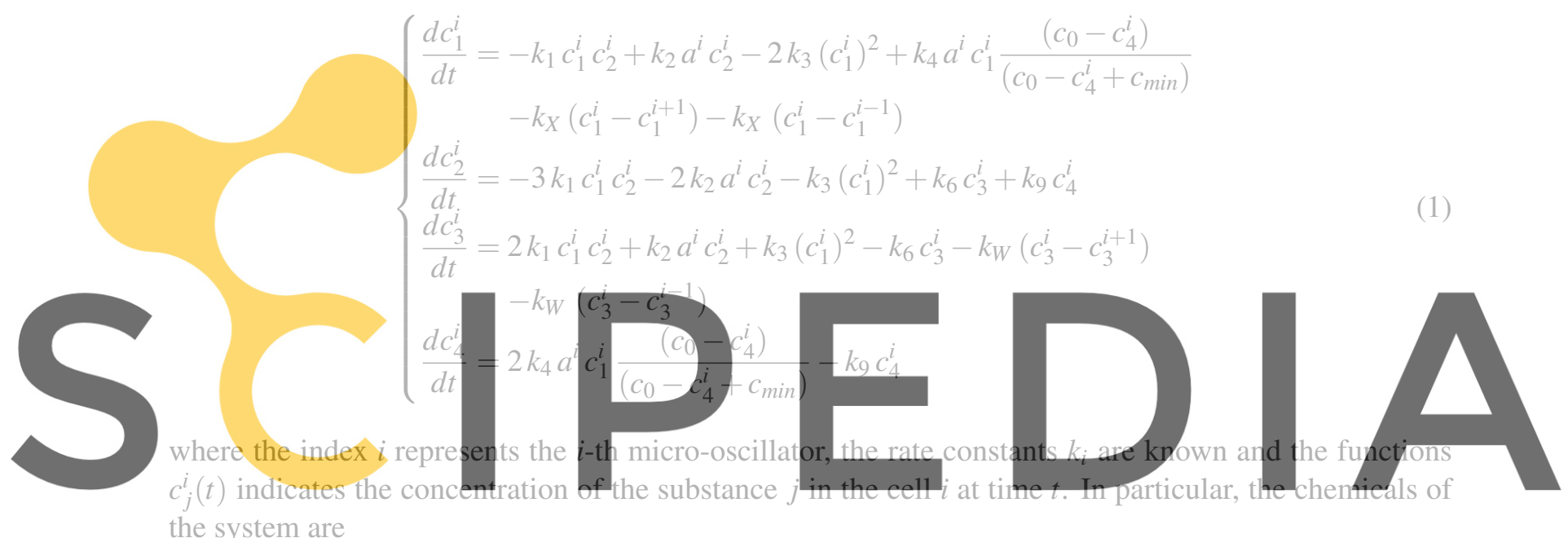

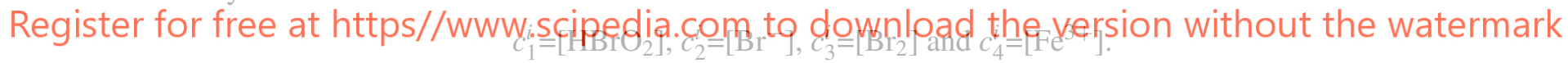

Other fixed and constant parameters of the system (1) are $c_{0}, c_{\min }$ and the coupling constants $k_{X}$ and $k_{W}$.

Finally, the vector $a$ has dimension equal to the number of droplets considered, and each component is used to modulate the natural frequency of the corresponding oscillator. Coupling terms $k_{X}\left(c_{1}^{i}-c_{1}^{j}\right)$ and $k_{W}\left(c_{3}^{i}-c_{3}^{j}\right)$ accounts for the intercellular communication between subsequent droplets mediated by the activator $\mathrm{HBrO}_{2}$ and the inhibitor $\mathrm{Br}_{2}$. The coupling intensity is given by the constants $k_{X}$ and $k_{W}$, which depend on compartmentalisation constraints, such as permeability or lamellarity of the membrane, which also account for the droplets distance.

Starting from the set of ODEs (1), in this work we insert a delay time $(\tau)$, which affect the coupling term of adjacent micro-oscillators. The corresponding DDEs set is reported in (2). To understand whether the new model improves the matching with experimental results, we will compare our simulations with the experiments reported in [5] previously simulated with ODEs (1). 


$$
\left\{\begin{aligned}
\frac{d c_{1}^{i}}{d t}= & -k_{1} c_{1}^{i} c_{2}^{i}+k_{2} a^{i} c_{2}^{i}-2 k_{3}\left(c_{1}^{i}\right)^{2}+k_{4} a^{i} c_{1}^{i} \frac{\left(c_{0}-c_{4}^{i}\right)}{\left(c_{0}-c_{4}^{i}+c_{\text {min }}\right)} \\
& -k_{X}\left(c_{1}^{i}-c_{1}^{i+1}(t)\right)-k_{X}\left(c_{1}^{i}-c_{1}^{i-1}(t)\right) \\
\frac{d c_{2}^{i}}{d t}= & -3 k_{1} c_{1}^{i} c_{2}^{i}-2 k_{2} a^{i} c_{2}^{i}-k_{3}\left(c_{1}^{i}\right)^{2}+k_{6} c_{3}^{i}+k_{9} c_{4}^{i} \\
\frac{d c_{3}^{i}}{d t}= & 2 k_{1} c_{1}^{i} c_{2}^{i}+k_{2} a^{i} c_{2}^{i}+k_{3}\left(c_{1}^{i}\right)^{2}-k_{6} c_{3}^{i}-k_{W}\left(c_{3}^{i}-c_{3}^{i+1}(t-\tau)\right) \\
& -k_{W}\left(c_{3}^{i}-c_{3}^{i-1}(t-\tau)\right) \\
\frac{d c_{4}^{i}}{d t}= & 2 k_{4} a^{i} c_{1}^{i} \frac{\left(c_{0}-c_{4}^{i}\right)}{\left(c_{0}-c_{4}^{i}+c_{\min }\right)}-k_{9} c_{4}^{i}
\end{aligned}\right.
$$

\section{NUMERICAL RESULTS}

The general form of the DDEs system (2) is

$$
\begin{cases}y^{\prime}(t)=f(t, y(t), y(t-\tau)) & t \geq t_{0} \\ y(t)=\phi(t) & t \leq t_{0}\end{cases}
$$

the history function $\phi(t)$ represents the value of the concentrations of substances involved in the DDEs

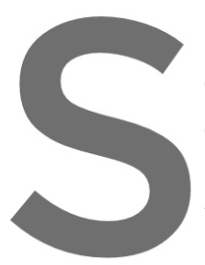
system (2) before the initio constant vector $y_{0}$. To coupled micro-oscillato and the phase difference at We employed several Ma
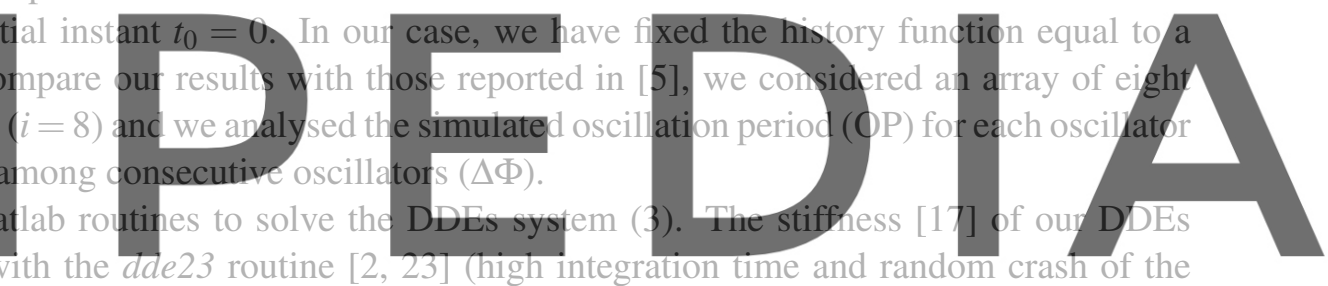

created some problems with the dde23 routine [2,23] (high integration time and random crash of the code). For this reason we finally employed the dde $15 \mathrm{~s}$ code, written by Dr. Lawrence F. Shampine

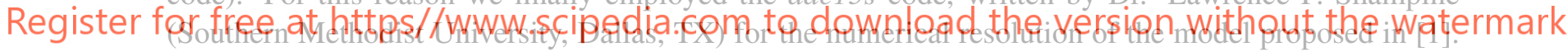

This numerical algorithm is based on the Matlab ode15s [22] routine for solving stiff ODEs systems; radar5 code [12] aiso yieids similar results.

As preliminary results, we simply explored the influence of the delay time on the oscillation parameters by fixing the initial conditions and all the constants and by varying $\tau$. Figures 1 and 2 show that, from an initial anti-phase synchronisation between consecutive oscillators, which occurs when there is no delay, an in-phase synchronisation when $\tau=110 \mathrm{~s}$ is achieved. Changes in the oscillation period of the system droplets and in the phase differences are shown in Figure 3. In this case, we can observe that, due to a very large delay, the oscillation period tends to lower by about 20/30 s compared to the case where there is no delay. Synchronization between consecutive oscillators is perfectly in-phase in the case with delay. With delays smaller than $\tau=110 \mathrm{~s}$, the numerical results are quite similar to the case when there is no delay.

\section{CONCLUSIONS}

At present, our preliminary results do not allow to make a direct comparison between the ODEs-based modelling (1) and the introduction of the delay time in (2). However, our analysis showed a significant 
Giovanni Pagano, Marcello Antonio Budroni, Raffaele D’Ambrosio, Dajana Conte, Ali Abou Hassan, Sandra Ristori, Federico Rossi and Beatrice Paternoster

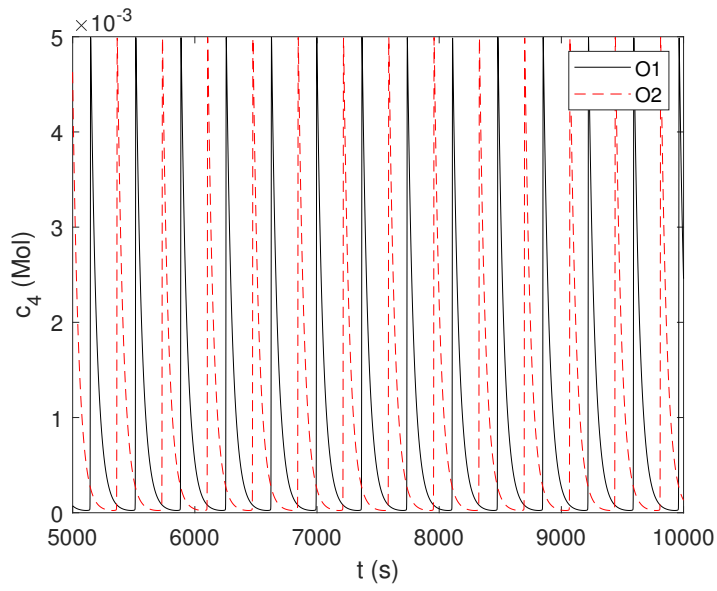

(a) MOs one and two with $\tau=0$

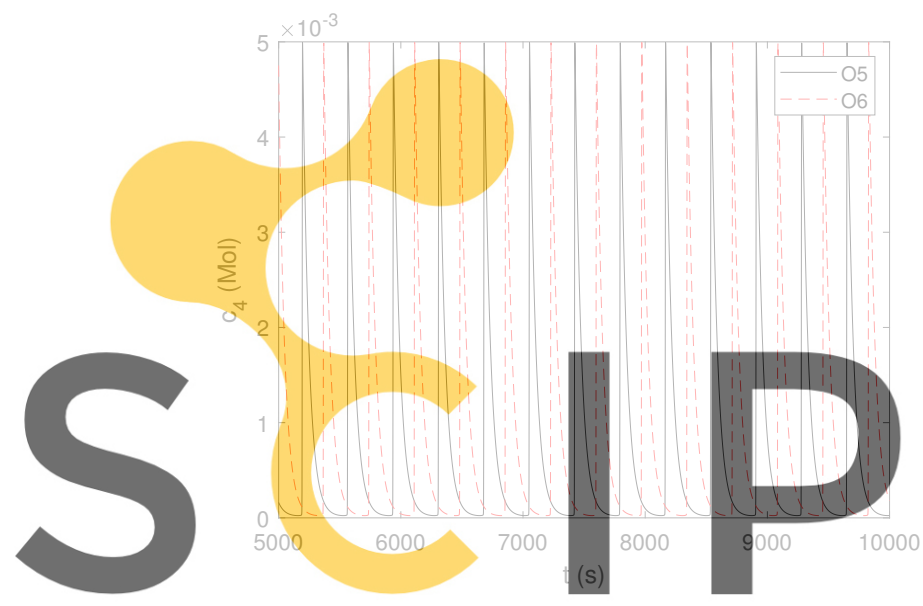

(c) MOs five and six with $\tau=0$

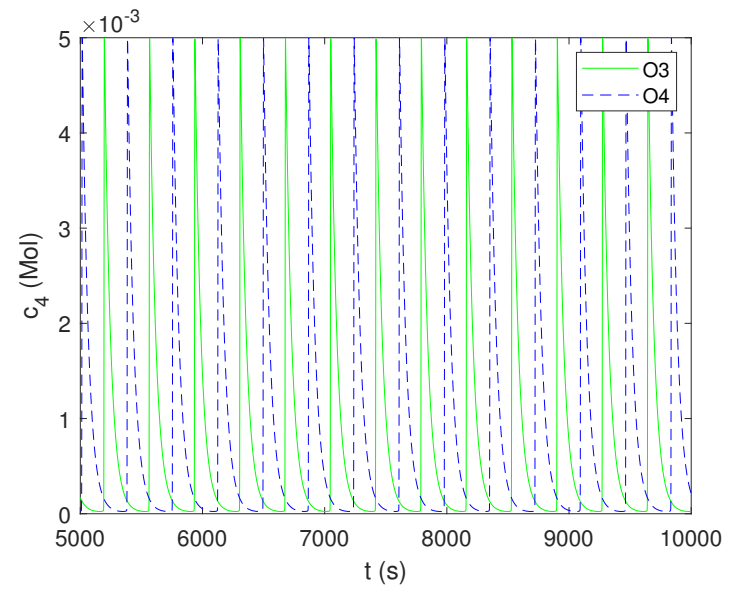

(b) MOs three and four with $\tau=0$

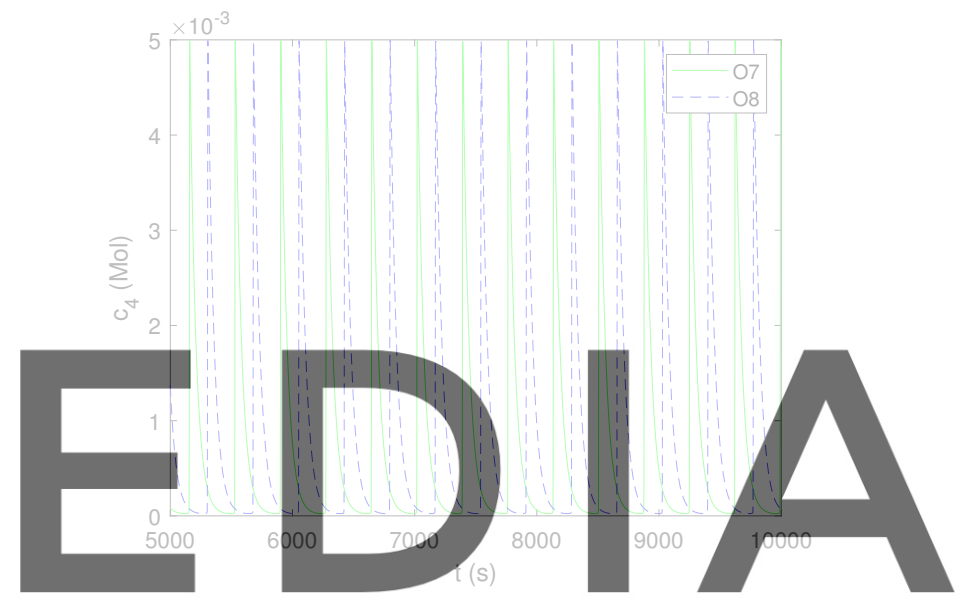

(d) MOs seven and eight with $\tau=0$

\section{Register for free at https//www.scipedia.com to download the version without the watermark Figure 1: Trend of $c_{4}$ for all eight MicroOscillators (MOs) of the system, with $\tau=0$.}

impact of $\tau$ on the global dynamics of the coupled micro-oscillators. In fact, by increasing the delay time, an initial anti-phase synchronisation scenario shifts to an in-phase configuration.

This observation is just a starting point for the ongoing investigation we are carrying out. A phase diagram of the system with respect to the modelling parameters is being mapped out. In addition, we are also assessing a more detailed parametrisation of the DDEs system with respect to the chemical properties of the molecules involved, this, for example, will suggest the insertion of a new different delay time for the activator species, now doesn't set.

Finally, we are also investigating in details the numerical resolution of the DDEs system (2) to overcome the stiffness issue. For example, we are adapting the works of D'Ambrosio et al. [7, 8], based on the EF technique, to consider the apriori known oscillating trend of the DDEs system solution. 
Giovanni Pagano, Marcello Antonio Budroni, Raffaele D’Ambrosio, Dajana Conte, Ali Abou Hassan, Sandra Ristori, Federico Rossi and Beatrice Paternoster

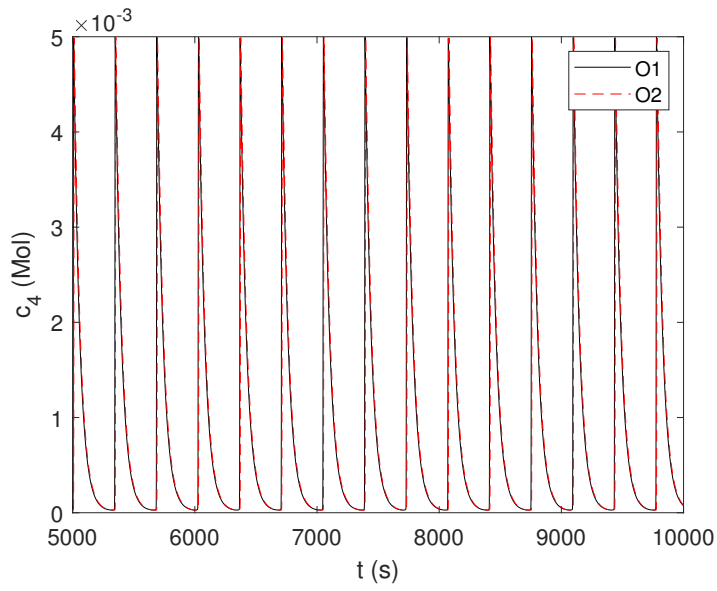

(a) MOs one and two with $\tau=110$

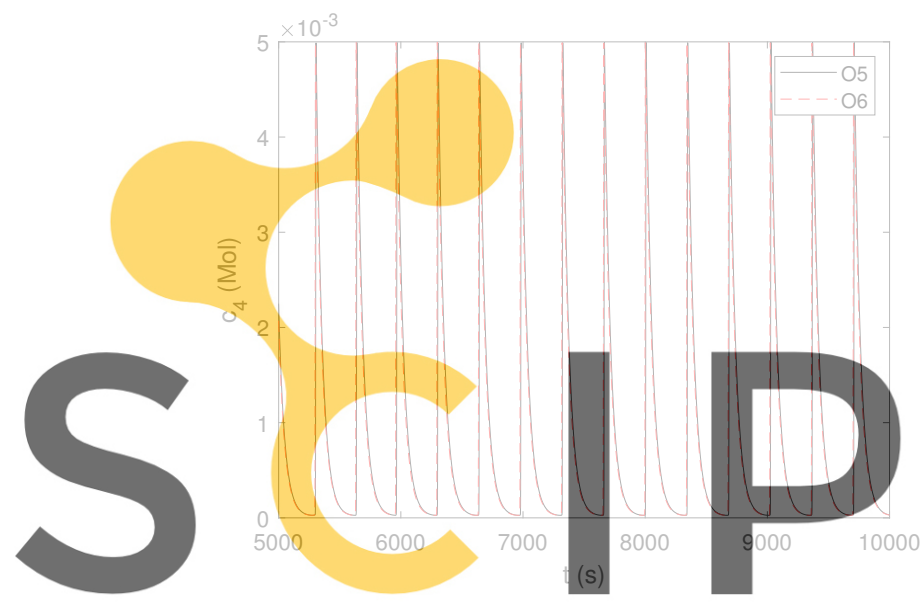

(c) MOs five and six with $\tau=110$

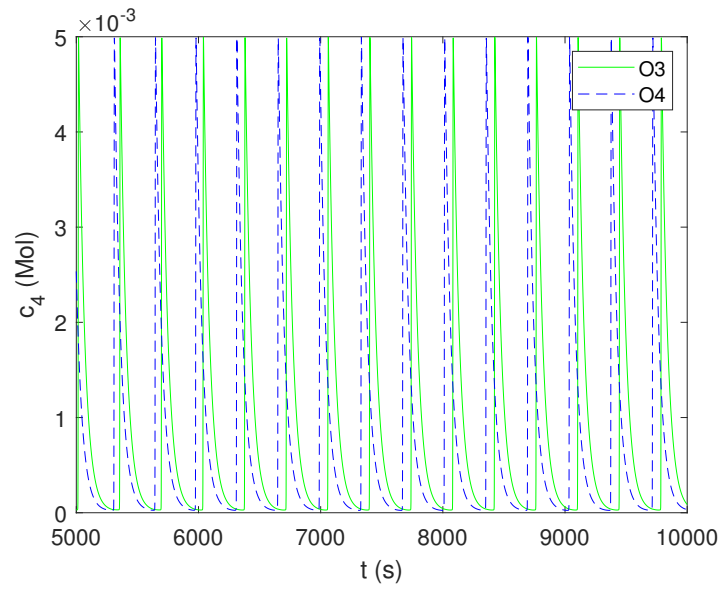

(b) MOs three and four with $\tau=110$

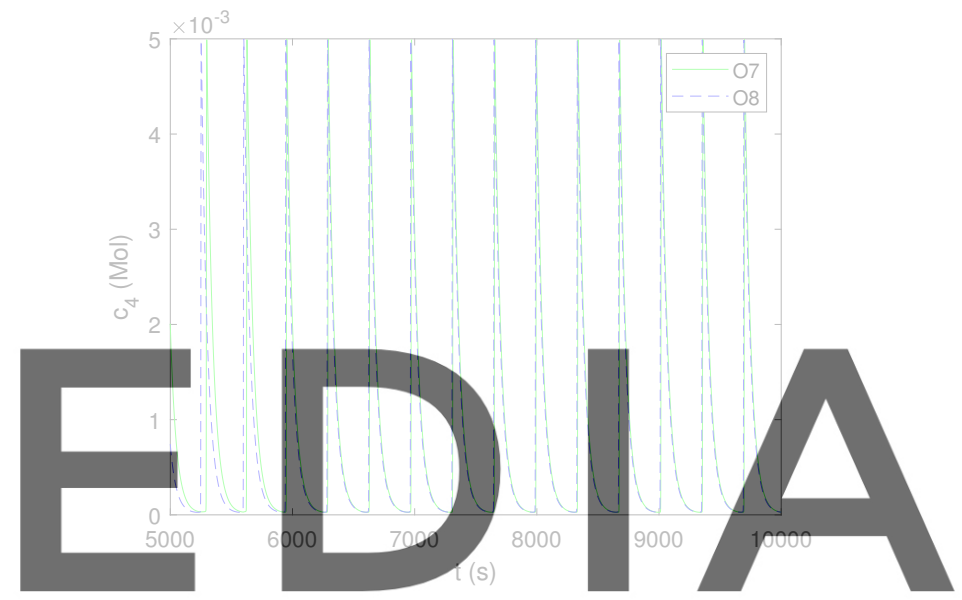

(d) MOs seven and eight with $\tau=110$

Register for free at https//www.scipedia.com to download the version without the watermark Figure 2: Trend of $c_{4}$ for all eight MicroOscillators (MOs) of the system, with $\tau=110$.

\section{REFERENCES}

[1] Agrawal, V., Zhang, C., Shapiro, A.D., Dhurjati, P.S. (2004). A dynamic mathematical model to clarify signaling circuitry underlying programmed cell death control in Arabidopsis disease resistance. Biotechnol. Prog., 20, 426-42.

[2] Bellen, A., Zerraro, M. (2003). Numerical Methods for Delay Differential Equations. Editor: Oxford University Press. Book necklace: Numerical Mathematics and Scientific.

[3] Belousov, B. P. (1959). An oscillating reaction and its mechanism. Sborn. referat. radiat. med. (Collection of abstracts on radiation medicine), Medgiz.

[4] Budroni, M.A., Rossi, F. (2015). A novel mechanism for in situ nucleation of spirals controlled by the interplay between phase fronts and reaction-diffusion waves in an oscillatory medium. J. Phys. Chem., C119(17), 9411-9417. 
Giovanni Pagano, Marcello Antonio Budroni, Raffaele D’Ambrosio, Dajana Conte, Ali Abou Hassan, Sandra Ristori, Federico Rossi and Beatrice Paternoster

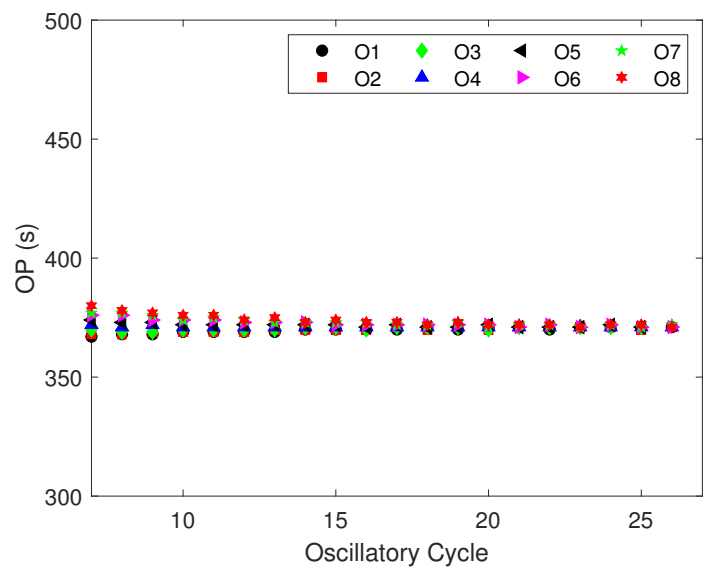

(a) Oscillation period with $\tau=0$

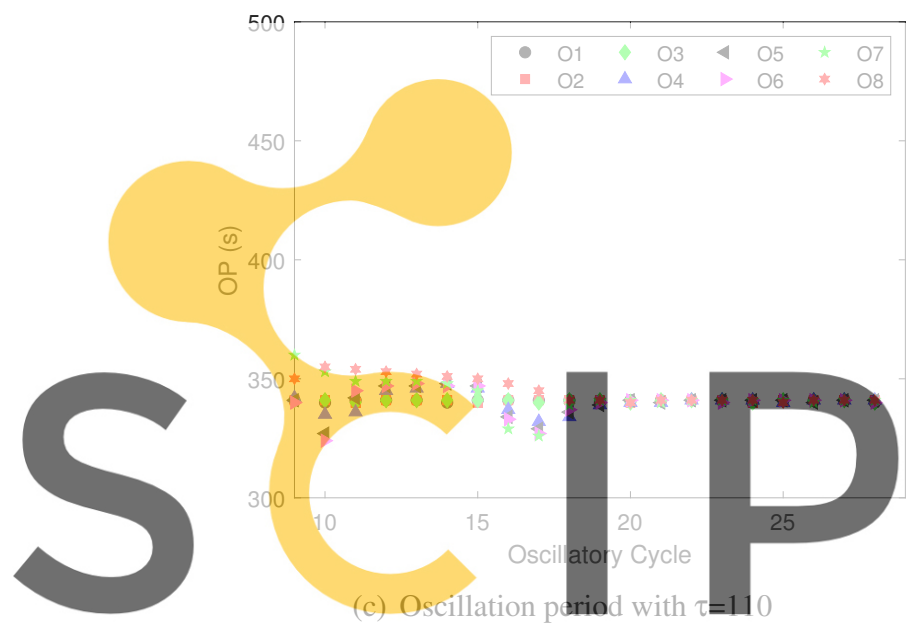

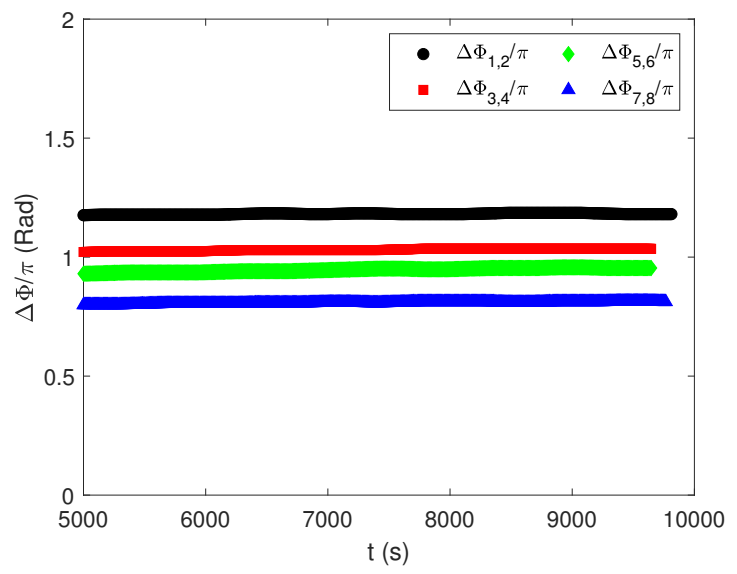

(b) Phase difference for successive MOs with $\tau=0$

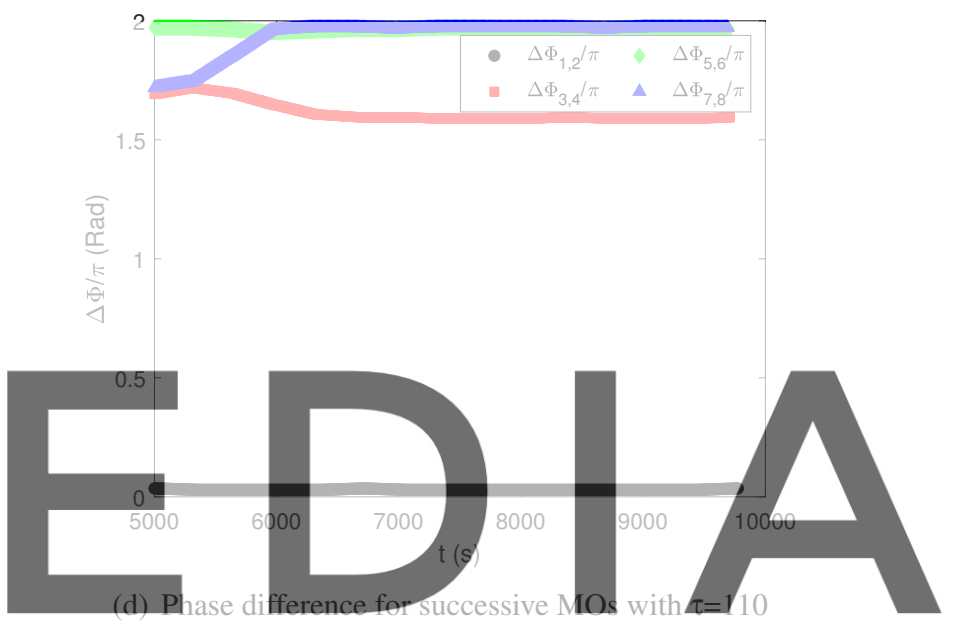

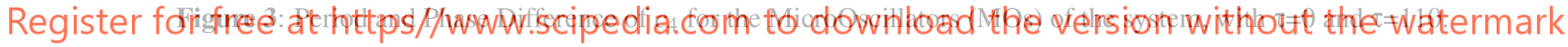

[5] Budroni, M.A., Torbensen, K., Ristori, S., Abou-Hassan, A., \& Rossi, F. (2020). Membrane Structure Drives Synchronization Patterns in Arrays of Diffusively Coupled Self-Oscillating Droplets. J. Phys. Chem. Lett., 11, 2014-2020.

[6] Budroni, M.A., Torbensen, K., Pantani O.L., Ristori, S., Rossi, F., \& Abou-Hassan, A. (2020). Microfluidic Compartmentalization of Diffusively Coupled Oscillators in Multisomes Induces a Novel Synchronization Scenario. Chem. Commun., 58(79), 11771-11774.

[7] D’Ambrosio, R., Moccaldi, M., Paternoster, B., Rossi, F. (2017). On the Employ of Time Series in the Numerical Treatment of Differential Equations Modeling Oscillatory Phenomena. Communications in Computer and Information Science, Vol. 708, 10.1007/978-3-319-57711-1-16.

[8] D’Ambrosio, R., Moccaldi, M., Paternoster, B., \& Rossi, F. (2018). Adapted numerical modelling of the Belousov-Zhabotinsky reaction. J. Math. Chem., 56, 2876-2897.

[9] Conte, D., D’Ambrosio, R., Pagano, G., Paternoster, B. (2020). Jacobian-dependent vs Jacobian- 
Giovanni Pagano, Marcello Antonio Budroni, Raffaele D’Ambrosio, Dajana Conte, Ali Abou Hassan, Sandra Ristori, Federico Rossi and Beatrice Paternoster

free discretizations for nonlinear differential problems. Computational and Applied Mathematics, 39. 10.1007/s40314-020-01200-z.

[10] Epstein I. R. and Xu, B. (2016). Reaction-diffusion processes at the nano- and microscales. Nat. Nano., 11, 312-319.

[11] Field, R. J. and Noyes, R. M. (1974). Oscillations in chemical systems. IV. Limit cycle behavior in a model of a real chemical reaction. J. Chem. Phys., 60, 1877-1884.

[12] Guglielmi, N., Hairer, E. (2001). Implementing Radau IIA Methods for Stiff Delay Differential Equations. Computing 67, 1-12. https://doi.org/10.1007/s006070170013.

[13] Ixaru, L. Gr. (2012). Runge-Kutta method with equation dependent coefficients. Comput. Phys. Commun., 183 (1):63-69.

[14] Ixaru, L. Gr. (2013). Runge-Kutta methods with equation dependent coefficients. Lect. Notes Comput. Sci., 8236:327-336.

[15] Ixaru, L. Gr. (2013). Runge-Kutta methods of special form. J. Phys. Conf. Ser., 413(1). Article number: 012033 .

[16] Ixaru, L. Gr. (2019). Exponential and trigonometrical fittings: user-friendly expressions for the coefficients. Numer. Algorithms, 82:1085-1096.

[17] Lambert, D. J. (1991). Numerical Methods for Ordinary Differential Systems: The Initial Value Problem. Editor: John Wiley and Sons Inc.
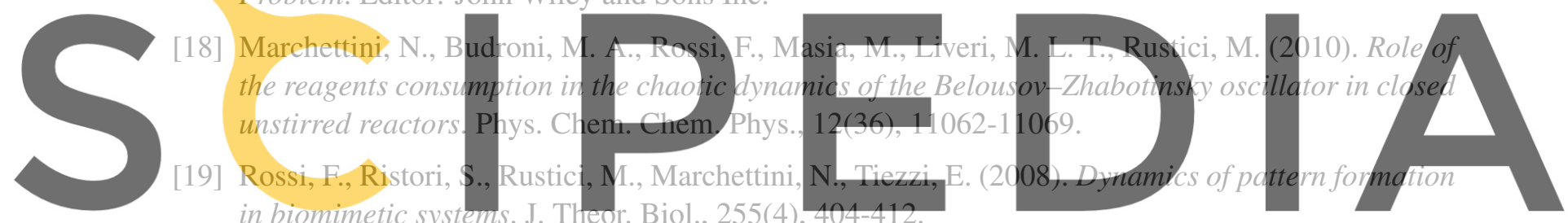
in biomimetic systems. J. Theor. Biol., 255(4),

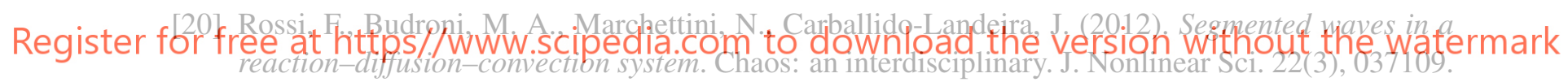

[21] Rossi F., Zenati, A., Ristori, S., Noel, J.-M., Cabuil, V., Kanoufi, F., Abou-Hassan, A. (2015). Activatory Coupling Among Oscillating Droplets Produced in Microfluidic Based Devices Int. J. Unconven. Comp., 11(1), 23-36.

[22] Shampine, L.F., Reichelt, M. (1997). The Matlab ODE suite. SIAM Journal on Scientific Computing, Vol. 18, No. 1, pp. 1-22, January 1997. 10.1137/S1064827594276424.

[23] Shampine, L. F., Gladwell, I., Thompson, S. (2003). Solving ODEs with MATLAB. Editor: Cambridge University Press.

[24] Taylor, A. F. (2002). Mechanism and phenomenology of an oscillating chemical reaction. Prog. React. Kinet. Mech. 27(4), 247-325.

[25] Tomasi, R., Noel, J.-M., Zenati, A., Ristori, S., Rossi, F., Cabuil, V., Kanoufi, F., Abou-Hassan, A. (2014). Chemical Communication between Liposomes Encapsulating a Chemical Oscillatory Reaction. Chem. Sci. 5(5), 1854-1859. 
Giovanni Pagano, Marcello Antonio Budroni, Raffaele D’Ambrosio, Dajana Conte, Ali Abou Hassan, Sandra Ristori, Federico Rossi and Beatrice Paternoster

[26] Torbensen, K., Rossi, F., Ristori, S., Abou-Hassan, A. (2017). Chemical communication and dynamics of droplet emulsions in networks of Belousov-Zhabotinsky micro-oscillators produced by microfluidics. Lab. Chip., 17(7), 1179-1189.

[27] Torbensen, K., Ristori, S., Rossi, F., Abou-Hassan, A. (2017). Tuning the chemical communication of oscillating microdroplets by means of membrane composition. J. Phys. Chem. C, 121(24), 1325613264.

[28] Zaikin, A.N., Zhabotinsky, A.M. (1970). Concentration wave propagation in two-dimensional liquid-phase self-oscillating system. Nature, 225(5232), 535-537.

[29] Zhabotinsky, A.M., Rossi, F. (2006). A brief tale on how chemical oscillations became popular: an interview with Anatol Zhabotinsky. Int. J. Des. Nat. Ecodyn., 1(4), 323-326.
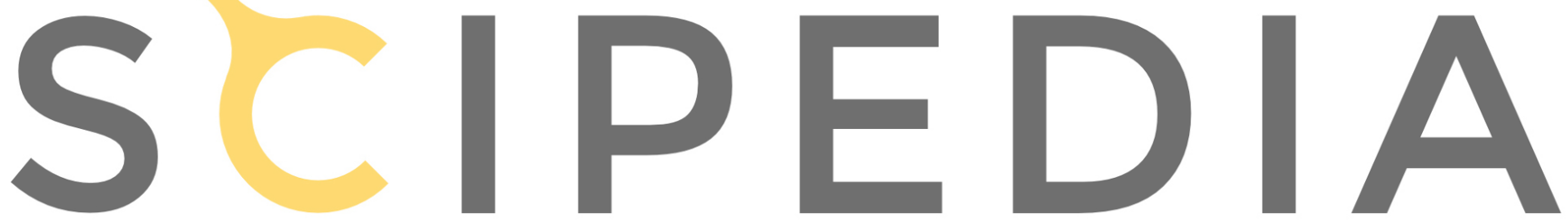

Register for free at https//www.scipedia.com to download the version without the watermark 